\title{
Tigecycline: A novel glycylcycline antibiotic
}

Tetracycline antibiotics were first isolated at Lederle Laboratories in 1945 and represented a significant advancement in the treatment of many infections. However, due to an increased incidence of resistance among various bacteria, the use of tetracyclines has been relegated to second and third-line categories for most clinical indications. The two major mechanisms of resistance include tetracycline efflux and ribosomal protection, where tetracycline is prevented from binding to the ribosome. Research to find tetracycline analogues, that circumvented these resistance mechanisms, has led to the development of a novel group of drugs called glycylcyclines, the most promising compound being the 9-tertbutyl glycyclamido derivative of minocycline-tigecycline (GAR936).

\section{Chemistry}

The nucleus consists of four linear fused tetracyclic rings and there is the addition of N, N-dimethylglycylamido (DMG) group at C-9 position of minocycline..$^{[1]}$

Certain structural features are important to maintain the activity of tigecycline. The basic nitrogen of the glycyl unit is essential for its activity. Replacement of tert-butylamino group with n-propyl or n-hexyl substitution did not result in any change in potency. Like tetracyclines, tigecycline also forms chelation complexes with divalent cations which include calcium, magnesium and iron, thereby resulting in food-drug and drug-drug interactions, thus influencing its anti-microbial and pharmacokinetic properties. ${ }^{[2]}$

\section{Mechanism of action}

Tigecycline binds reversibly to the $30 \mathrm{~S}$ sub-unit of the bacterial ribosome, blocking the binding of amino-acyl-tRNA to the acceptor site on the mRNA-ribosome complex. This prevents the incorporation of amino acids to the growing peptide chain, thereby inhibiting protein synthesis.

The major mechanisms of resistance to tetracyclines include:

1. Decreased accumulation as a result of acquisition of energy dependent efflux pathway.

2. Production of a ribosomal protection protein via expression of tet $(\mathrm{M})$ gene that displaces tetracycline from

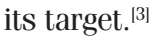

Resistance occurs through the acquisition of tetracycline resistance genes. Tigecycline has demonstrated anti-bacterial activity against tetracycline-resistant organisms which have tet $(\mathrm{M})$-protected ribosomes. They bind more avidly to the ribosomes and in a different orientation from classical tetracyclines ${ }^{[4]}$ so that the product of tet $(\mathrm{M})$ gene is unable to disrupt the tight bond, or that the product of tet $(\mathrm{M})$ gene is unable to interact with the ribosome to allow protein synthesis to occur. ${ }^{[5]}$ Tigecycline is also active against organisms that display efflux-based resistance, which may be because of the inability of the glycylcyclines to induce tetracycline efflux proteins, or because the efflux protein cannot export tigecycline. ${ }^{[6]}$

The binding site of tigecycline on the ribosome is common to tetracyclines, but tigecycline binds 5-fold more strongly to the ribosome than tetracyclines and this enhanced binding is, probably, responsible for overcoming the ribosomal protection mechanisms of tetracycline resistance. ${ }^{[5]}$ The action of tigecycline is bacteriostatic in nature, which is likely due to its reversible interaction with the ribosome. ${ }^{[5]}$ Its efficacy suggests that traditional thinking about using bacteriostatic drugs in treating serious infections needs to be revised. ${ }^{[7]}$

\section{Antimicrobial activity}

In vitro antibacterial activity of tigecycline has been assessed against clinical isolates as a part of ongoing TEST initiative (Tigecycline Evaluation Surveillance Trial).

The activity of tigecycline against staphylococci is completely unaltered by the presence of methicillin or glycopeptide resistance genes and remains fully effective against enterococci, expressing one or more vancomycin resistance determinants. Hence, tigecycline is highly effective against these organisms, namely methicillin resistant Staphylococcus aureus (MRSA), vancomycin resistant enterococci (VRE), and penicillin resistant Streptococcus pneumoniae. It also has activity against methicillin susceptible $S$. aureus, vancomycin susceptible enterococci, and penicillin susceptible $S$. pneumoniae. It is the most potent antimicrobial when tested against glycopeptide-intermediately resistant $S$. aureus (GISA). ${ }^{[8]}$

Tigecycline shows high potency against gram-negative bacilli such as Acinetobacter baumannii, Stenotrophomonas maltophilia and Klebsiella pneumoniae, whose multi-drug resistant strains have emerged as important nosocomial pathogens. Tigecycline is also active against clinically relevant species of Enterobacteriaceae, including extended-spectrum beta-lactamase (ESBL) producing strains..$^{\left[{ }^{\mid \rho}\right.}$ However, recently drug resistance has been reported in Proteus miribalis and Pseudomonas aeruginosa strains due to its extrusion by multidrug efflux pumps. ${ }^{[10]}$ Tigecycline's expanded, broad-spectrum activity is further evidenced by its activity against Legionella pneumophilia, Chlamydia, rapidly growing non-tuberculosis bacteria and various anaerobes such as Nocardia, Bacteroides and Clostridia species. ${ }^{[1]}$

\section{Pharmacokinetics}

Tigecycline is currently available only for intravenous (i.v.) use. 
After iv administration, tigecycline exhibits linear pharmacokinetics after single dose in the range of 12.5 to $300 \mathrm{mg}$ and multiple doses of 25 to $100 \mathrm{mg}$, every $12 \mathrm{~h}$.

Tigecycline is extensively distributed into various tissues and achieves high drug concentration in the organs, including lung, skin, liver, heart and particularly, bones. This suggests that measurement of its plasma concentration may significantly underestimate the concentration of the drug in various tissues.

Broad tissue penetration of tigecycline is evidenced by its high volume of distribution of more than $10 \mathrm{~L} / \mathrm{kg}$, following the administration of a $100 \mathrm{mg}$-loading dose, followed by twicedaily doses of $50 \mathrm{mg}$. Its plasma protein binding ranges from $71 \%$ to $87 \%$ at plasma concentration of 0.1 and $1 \mu \mathrm{g} / \mathrm{ml}$, respectively. It has a long terminal elimination half-life of about $36 \mathrm{~h}$ which, allows for twice-daily dose administration. The elimination of tigecycline was slower from tissues than from plasma, yielding high tissue to plasma ratios, particularly in the bone marrow, thyroid, spleen and liver. ${ }^{[12]}$

No major metabolites have been found to date. The major mode of its excretion in humans appears to be through the biliary route. Less than $15 \%$ of tigecycline is excreted, unchanged, in urine.

The pharmacokinetics of tigecycline are unaffected by food, age, gender, renal disease or hepatic disease..$^{[7]}$

Tigecycline exhibits time-dependent antimicrobial activity. The pharmacodynamic parameter that best correlates with bacteriological eradication is time above minimum inhibitory concentration. ${ }^{[1]}$ For maximum therapeutic efficacy, the concentration of tigecycline should be maintained above the MIC for at least $50 \%$ of the dose-interval. ${ }^{[11]}$ The post antibiotic effect of tigecycline was 8.9 hours against $S$. pneumoniae, 4.9 hours against Escherichia coli and $3 \mathrm{~h}$ against $S$. aureus. ${ }^{[11]}$

Clinical trials have shown that tigecycline $(50 \mathrm{mg}$, i.v. every $12 \mathrm{~h}$ ) in adults is safe and generally well tolerated for upto 11.5 days. $^{[13]}$

\section{Clinical trials}

Two pairs of similarly designed, non-inferiority, activecomparator controlled, Phase III clinical trials were undertaken for licensing: one trial comparing tigecycline with aztreonam plus cilastatin in complicated intra-abdominal infections (cIAI), and the other comparing tigecycline with aztreonam plus vancomycin in complicated skin and skin structure infections (cSSI). Over 2850 patients were treated in these trials concluding that tigecycline is no less effective than the active

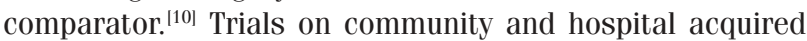
pneumonias are in progress and, if positive, will form the basis of a license extension. ${ }^{[10]}$ In addition, there are ongoing trials against infections caused by specific multi-resistant pathogens, along with an extensive compassionate-use programme. ${ }^{[10]}$

\section{Indications}

Tigecycline can be used as an empiric monotherapy to treat a variety of serious bacterial infections, both hospital and community acquired, including complicated appendicitis, infected burns, intra-abdominal abscesses, deep soft tissue infections and infected ulcers. It is administered as an intravenous infusion over 30-60 min as $100 \mathrm{mg}$ (loading dose), followed by $50 \mathrm{mg}$ every $12 \mathrm{~h}$ (maintenance dose) for 5-14 days. $^{[14]}$ In case of hepatic insufficiency, maintenance dose should be reduced to $25 \mathrm{mg}$. ${ }^{[14]}$

\section{Current status}

Tigecycline is not yet available in India. US FDA has approved tigecycline for treatment of cSSI and cIAI in adults in June 2005. ${ }^{[14]}$ It is currently under review by regulatory agencies worldwide for other indications as well.

\section{Adverse effects}

The most frequent adverse events are gastrointestinal nausea (29.5\%) and vomiting (19.7\%). But these are mild to moderate in severity, of limited duration and do not usually require discontinuation of therapy. ${ }^{[13]}$ Tolerability of tigecycline in fasting subjects is improved by the use of anti-emetics. ${ }^{[13]}$ Clostridium difficile related complications with tigecycline are uncommon. ${ }^{[13]}$

\section{Caution}

Tigecycline may cause foetal harm when administered to pregnant women. The safety and effectiveness of tigecycline in patients below 18 years and lactating women has not been established. The use of tigecycline during tooth development may cause permanent discoloration of teeth. ${ }^{[13]}$

It should be administered with caution in patients with known hypersensitivity to tetracycline class antibiotics as it may have similar adverse effects. ${ }^{[13]}$

\section{Contraindication}

Tigecycline is contraindicated in patients with known hypersensitivity to tigecycline. ${ }^{[13]}$

\section{Conclusion}

Antibiotic development has slowed and only a few broadspectrum antibiotic agents are currently in development. Hence, new classes of antibiotics are urgently needed to address the increasing antibiotic resistance among common pathogens. For patients with serious infections, the initial choice for empirical therapy with broad spectrum antibiotics is crucial, and, if the choice is inappropriate, it may have adverse consequences for the patient.

Tigecycline, a novel, broad-spectrum potential glycylcycline, has been shown to be active against many of gram-positive, gram-negative, atypical and anaerobic organisms. These include highly resistant strains of clinical importance such as community and hospital acquired MRSA, VRE, penicillin resistant $S$. pneumoniae and ESBL expressing E. coli and K. pneumoniae. Tigecycline has come into clinical use at a critical time and will be a welcome asset to the current armamentarium. Unlike tetracyclines, tigecycline does not require dose adjustment in patients with impaired renal function and is conveniently administered every $12 \mathrm{~h}$.

Since it has proven activity against highly resistant organisms, it should be reserved only for life-threatening situations and/or when resistant pathogens are suspected. Rational antimicrobial use, coupled with awareness of infection control measures, is paramount to avert the emergence of multi-drug resistant organisms.

Oral formulation of the drug would further expand the potential role of tigecycline therapy in clinical practice. 
K. Gupta, S. Kaushal, S. C. Chopra Department of Pharmacology,

Dayanand Medical College and Hospital, Ludhiana - 141001, Punjab. India E-mail: skaushal1@yahoo.co.in

\section{References}

1. Zhanel GG, Homenuik K, Nichol K. The glycylcyclines: A comparative review with the tetracyclines. Drugs 2004;64:63-88.

2. Chopra I, Roberts M. Tetracycline antibiotics: Mode of action, applications, molecular biology, and epidemiology of bacterial resistance. Microbiol Mol Biol Rev 2001;65:232-60.

3. Chambers HF. Protein synthesis inhibitors and miscellaneous antibacteria agents. Brunton LL, Lazo JS, Parker KL, eds. In: Goodman and Gilman's The Pharmacological Basis of Therapeutics. 11 ${ }^{\text {th }}$ ed. New York: McGraw-Hill; 2006. p. 1174.

4. Bauer G, Berens C, Projan SJ, Hillen W. Comparison of tetracycline and tigecycline binding to ribosomes mapped by dimethylsulphate and drug-directed $\mathrm{Fe}^{2+}$ cleavage of 16S rRNA. J Antimicrob Chemother 2004;53:592-9.

5. Bergeron J, Ammirati M, Danley D. Glycylcyclines bind to the high-affinity tetracycline ribosomal binding site and evade Tet $(\mathrm{M})$, -and Tet $(\mathrm{O})$-mediated ribosomal protection. Antimicrob Agents Chemother 1996;40:2226-8.
6. Someya Y, Yamaguchi A, Sawai T. A novel glycylcycline, 9-(N,Ndimethylglycylamido)-6-demethyl-6-deoxytetracycline, is neither transported not recognized by the transposon $\mathrm{Tn} 10$-encoded metal-tetracycline/ $\mathrm{H}+$ antiporter. Antimicrob Agents Chemother 1995;39:247-9.

7. Nathwani D. Tigecycline: Clinical evidence and formulary positioning. Int $\mathrm{J}$ Antimicrob Agents 2005;25:185-92.

8. Felmingham D. Tigecyclin: The first glycylcycline to undergo clinical development: an overview of in vitro activity compared to tetracycline. J Chemother 2005;17:5-11

9. Meagher AK, Ambrose PG, Grasela TH, Ellis-Grosse EJ. Pharmacokinetic/ pharmacodynamic profile for tigecycline: A new glycylcycline antimicrobial agent. Diagn Microbiol Infect Dis 2005;52:165-71.

10. Livermore DM. Tigecycline: what is it and where should it be used? J Antimicrob Chemother 2005;56:611-4.

11. Van Ogtrop ML, Andes D, Stamstad TJ. In vivo Pharmacodynamic activities of two glycylcylines (GAR-936 and WAY-152,288) against various gram-positive and gram-negative bacteria. Antimicrob Agents Chemother 2000;44:943-9.

12. Tombs NI. Tissue distribution of GAR-936, a broad spectrum antibiotic, in male rats [abstract no. 413]. 39 Interscience Conference on Antimicrobial Agents and Chemotherapy. San Francisco: 1999.

13. Rello J. Pharmacokinetics, pharmacodynamics, safety and tolerability of tigecycline. Chemotherapy 2005;17:12-22.

14. Frampton JE, Curran MP. Tigecycline. Drugs 2005;65:2023-35.

\section{Unleash the power of your literature search}

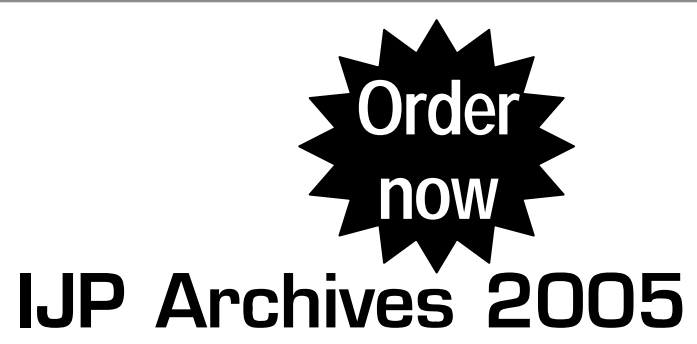

\section{Rs. 500/- for IPS members}

Rs. 2000/- for Libraries \& Educational Institutes in India
O Full articles in PDF format from 1969-2005
O Versatile search facility
O Easy installation
O User friendly interface
In-built help and instructions

For details please contact:

The Chief Editor - IJP

Department of Pharmacology,

JIPMER, Pondicherry-605 006.

E-mail: ijp@jipmer.edu 